\title{
An Efficient and Sustainable Approach to Prepare Carboxylated Cellulose Nanocrystals for Rubber Reinforcement Featuring Dual Crosslinking Networks
}

Jie Hu

Qingdao University of Science and Technology

Zhengqing Kong

Qingdao University of Science and Technology

Ke Liu

Qingdao University of Science and Technology

Jinli Qin

Qingdao University of Science and Technology

Yuhong Tao

Qingdao University of Science and Technology

Lijuan Zhou

Qingdao University of Science and Technology

Yuan Yuan

Qingdao University of Science and Technology

Min Jiang

Qingdao University of Science and Technology

Yongxin Duan ( $\nabla$ dyx@qust.edu.cn )

Qingdao University of Science and Technology

https://orcid.org/0000-0003-3075-5730

Jianming Zhang

Qingdao University of Science and Technology

\section{Research Article}

Keywords: Carboxylation, CNCs, XSBR, Nanocomposites, Dual crosslinking networks

Posted Date: June 18th, 2021

DOl: https://doi.org/10.21203/rs.3.rs-585397/v1

License: (9) This work is licensed under a Creative Commons Attribution 4.0 International License. Read Full License 
Page $2 / 21$ 


\section{Abstract}

The surface functionalization of CNCs and the construction of strong interfacial adhesion between CNCs and rubber matrix are effective way to achieve high performance rubber/CNCs nanocomposites. Herein, carboxylation of sulphated cellulose nanocrystals $\left(\mathrm{CNC}-\mathrm{OSO}_{3} \mathrm{H}\right)$ was conducted in aqueous medium by using citric acid as modifier. Large amount of carboxyl groups was successfully grafted on the surface of $\mathrm{CNC}-\mathrm{OSO}_{3} \mathrm{H}$, which endows the carboxylated $\mathrm{CNC}-\mathrm{OSO}_{3} \mathrm{H}$ (abbreviate as $\mathrm{CNC}-\mathrm{CA}$ ) with higher chemical reactivity and thermal stability. Subsequently, carboxylated styrene butadiene rubber (XSBR)/CNC-CA nanocomposites with dual crosslinking design were prepared by using polyethylene glycol diglycidyl ether (PEGDE) as the crosslinking agent and CNC-CA as the reinforcing fillers. FTIR investigation found that in the obtained nanocomposites, the carboxyl groups on CNC-CA and XSBR formed hydrogen bonds (physical crosslinking) with each other, and the carboxyl groups formed covalent bond with the epoxy group on PEGDE simultaneously. The coexistence of physical and chemical crosslinking improved the interface compatibility between CNC-CA and XSBR matrix, accelerated the homogenous dispersion of CNC-CA and realized the crosslinking of the matrix itself. As expected, XSBR/CNC-CA nanocomposites with dual crosslinking network showed remarkable enhancement in tensile strength (up to $500 \%$ ), modulus (up to $151 \%$ ), work of fracture (up to $348 \%$ ). This work provides both a facile and green approach to obtain carboxylated CNCs and a convenient method for the preparation of high-performance rubber nanocomposites with multiple interactions.

\section{Introduction}

By loading reinforcing fillers, the mechanical properties of composites can be improved largely compared to the neat matrix (Bitinis et al. 2011; Chen et al. 2008; Ramorino et al. 2009; Rooj et al. 2010). With the development of nanoscience, the application of various nano-reinforcing fillers in the field of composite materials has attracted increasing attention (Ding et al. 2019; Iwatake et al. 2008; Potts et al. 2012; Sengupta et al. 2011; Tang et al. 2012; Wu et al. 2018). Cellulose is the most abundant biopolymer resource on the planet and has some nanosized and hierarchical structure. As one kind of nanocellulose, cellulose nanocrystals (CNCs) are considered as a promising "green" filler for composite materials owing to the various advantages, such as large specific surface area, high tensile strength, high aspect ratio, high Young's modulus, plenty of surface hydroxyl groups, biocompatibility and biodegradability (Kargarzadeh et al. 2018; Klemm et al. 2018; Thomas et al. 2018; Dufresne et al. 2018; Habibi et al. 2010). In 2011, the preparation of CNCs was industrialized via sulfuric acid hydrolysis, and this further promotes the application of CNCs in the field of composite materials. The CNCs prepared by this method have sulfonate groups on the surface and is abbreviated as $\mathrm{CNC}^{-} \mathrm{OSO}_{3} \mathrm{H}$. Due to the merits of fast reaction speed, simple process, high yield and good dispersion of $\mathrm{CNC}-\mathrm{OSO}_{3} \mathrm{H}$ in aqueous solution, sulfuric acid hydrolysis is the only industrialized preparation method at present. CNCs were first adopted to reinforce rubber in 1995 by Favier et. al, since then plenty of reports about rubber/CNCs composites emerge (Eslami et al. 2020; Flauzino Neto et al. 2016; Jiang and Gu 2020; Kargarzadeh et al. 2017; Nunes 2017; Parambath Kanoth et al. 2015; Peng et al. 2018; Peng et al. 2019; Tian et al. 2017; Favier et al. 1995). 
Rachel Blanchard et. al reported that the incorporation of $\mathrm{CNC}^{-} \mathrm{OSO}_{3} \mathrm{H}$ effectively improved the crosslinking density, tensile strength and modulus of the rubber latex for the application in dipped goods (Blanchard et al. 2020). However, the poor heat resistance of $\mathrm{CNC}^{-} \mathrm{OSO}_{3} \mathrm{H}$ caused by sulfonate group and relatively low chemical reactivity remain a problem which cannot be ignored (Jiang et al. 2010;Wang et al.

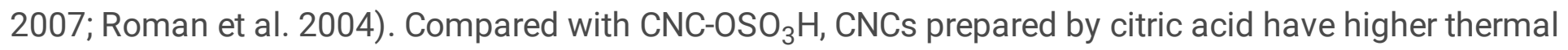
stability, and the surface carboxyl groups endow CNCs higher reactivity. Yet, due to the weak acidity of citric acid, the yield of CNCs prepared by citric acid alone is quite low (Ji et al. 2019). A large amount of citric acid is needed when mixed with other inorganic acids to prepare CNCs, and it is not only the preparation process is time-consuming but also difficult to realize the recycling of citric acid (Yu et al. 2016).

Carboxylated styrene butadiene rubber (XSBR), a copolymer of styrene, butadiene and a small amount of

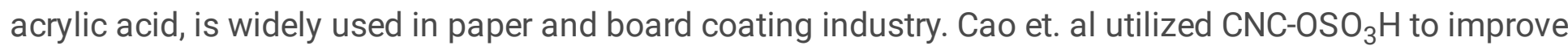
the mechanical properties of XSBR matrix by XSBR latex/CNC-OSO${ }_{3} \mathrm{H}$ co-sedimentation and two-roll mill blending (Cao et al. 2013). Even though the addition of $\mathrm{CNC}-\mathrm{OSO}_{3} \mathrm{H}$ improved the mechanical properties of XSBR/CNC-OSO ${ }_{3} \mathrm{H}$ nanocomposites to a certain extent, there are still some unsolved problems: the poor thermal stability of $\mathrm{CNC}-\mathrm{OSO}_{3} \mathrm{H}$, the agglomeration of $\mathrm{CNC}-\mathrm{OSO}_{3} \mathrm{H}$ in matrix and inadequate interaction between fillers-rubber (Chen et al. 2015). In order to improve the compatibility and interfacial strength between CNCs and matrix, a lot of efforts have been made. Cao et al. used maleic anhydride grafted tunicate cellulose nanocrystals ( $t-C N s$ ) to reinforce the epoxidized natural rubber by the construction of dual cross-linked network. The interfacial chemical reaction between carboxyl groups on modified t-CNs and epoxy groups on ENR chains formed covalent crosslinking network, and the hydrogen bonds between hydroxyl groups on t-CNs and epoxy groups on ENR chains served as physical crosslinking network. It is worth mentioning that, even though the covalent bond formed between modified $\mathrm{t}-\mathrm{CN}$ s and ENR chains effectively improve the interface compatibility and adhesion between filler and matrix, the uncrosslinking matrix still leads to the limited improvement of the mechanical properties of the composites (Cao et al. 2018). Tao et al. designed a novel double-network composite system of reducing end-modified CNCs ( $\mathrm{mCNC}$ ) enhanced thermoplastic elastomer with click reaction and bulk crosslinking. Though, the combination of filler-matrix, filler-filler and matrix-matrix interaction effectively realized the mechanical enhancement of the composites, the modification process of CNCs and the preparation of composites were quite tedious (Tao et al. 2019). The above works demonstrate that the construction of dual crosslinking network is an effective way to improve the properties of rubber composites. As the reactive functional carboxyl group of XSBR provides the possibility for the formation of hydrogen bond and covalent bond between XSBR and reinforcing fillers. The key point is to activate and desulfonate the surface of $\mathrm{CNC}-\mathrm{OSO}_{3} \mathrm{H}$, subsequently design a strong XSBR-CNCs and XSBR-XSBR interaction network.

In this work, $\mathrm{CNC}-\mathrm{OSO}_{3} \mathrm{H}$ were firstly carboxylated by reaction with citric acid in aqueous phase under a mild condition, the reaction solvent and ethanol used for centrifugal washing could be recycled. Then XSBR/CNC-CA nanocomposites with dual crosslinking network were constructed by using polyethylene 
glycol diglycidyl ether (PEGDE) as crosslink agent and 2-methylimidazole (2-mlm) as catalyst. FTIR investigation confirmed that the hydrogen bonds (physical crosslinking) formed among the carboxyl groups on CNC-CA and XSBR, the covalent bonds (chemical crosslinking) formed between the carboxyl groups and the epoxy group of PEGDE. Benefiting from the dual crosslinking structure, the interface compatibility between CNC-CA and XSBR matrix is improved, the homogenous dispersion of CNC-CA is accelerated and the crosslinking of the matrix itself is realized. Compared with XSBR/CNC-CA nanocomposites without PEGDE crosslinking, the tensile strength, modulus and work of fracture of XSBR/CNC-CA nanocomposites with PEGDE crosslinking are significantly improved.

\section{Experimental}

\section{Materials}

XSBR latex with 50 wt \% solid content was obtained from Shandong Xing Yu gloves Co., Ltd. (Gaomi, China). Cotton linter pulps (CLP) were provided by Silver Hawk Co. Ltd. (Gaomi, China). Citric acid anhydrous (CA, 99.5\%) was purchased from Sinopharm Chemical Reagent Co., Ltd. (Shanghai, China). Polyethylene glycol diglycidyl ether (PEGDE, 99\%) was purchased from Macklin reagent company (Shanghai, China). 2-methylimidazole (2-mlm, 98\%) was purchased from Aladdin Industrial Corporation (Shanghai, China). Sodium hydroxide, sulfuric acid, toluene and dimethylformamide are general commercial products. All regents are analytical grade.

\section{Preparation of sulfonic cellulose nanocrystals $\left(\mathrm{CNC}-\mathrm{OSO}_{3} \mathrm{H}\right)$}

$\mathrm{CNC}-\mathrm{OSO}_{3} \mathrm{H}$ was extracted from CLP via sulfuric acid hydrolysis as described in our previous work (Wang et al. 2020). In brief, raw CLP was first immersed in $4 \mathrm{wt} \% \mathrm{NaOH}$ solution for $24 \mathrm{~h}$ at room temperature. Then, the obtained slurry was thoroughly washed and dried in a vacuum oven. After that, the pretreated CLP was hydrolyzed in $64 \mathrm{wt} \%$ sulfuric acid at $50^{\circ} \mathrm{C}$ with continuous vigorous stirring, a large amount of water was used to dilute the mixture and terminate the acid hydrolysis process. Finally, the suspension was centrifuged repeatedly and washed until the $\mathrm{pH}$ of the centrifugal solution was close to 3 , followed by dialyzing against deionized water until neutral $\mathrm{pH}$ was achieved. The resultant $\mathrm{CNC}-\mathrm{OSO}_{3} \mathrm{H}$ suspension was concentrated to ca. $2 \mathrm{wt} \%$ by rotary evaporation at $40^{\circ} \mathrm{C}$, and carefully collected for the following experiment.

\section{Preparation of citric acid grafting CNCs (CNC-CA)}

First, $20 \mathrm{ml}$ of $\mathrm{CNC}-\mathrm{OSO}_{3} \mathrm{H}$ aqueous solution $(20 \mathrm{mg} / \mathrm{ml})$ was added to a flask and ultrasonic treated for $10 \mathrm{~min}$, then $10 \mathrm{~g}$ anhydrous CA was added to the flask and ultrasonic treated for another $10 \mathrm{~min}$. Subsequently, the flask was assembled to a distillation device, and the oil bath temperature was set at $120^{\circ} \mathrm{C}$. The water in the mixture was gradually evaporated with oil temperature increases. After the oil bath temperature reached $120^{\circ} \mathrm{C}$, the $\mathrm{CNC}-\mathrm{OSO}_{3} \mathrm{H}$ surface esterification was performed for $15 \mathrm{~h}$. After the reaction, the obtained CNC-CA was purified from unreacted CA by 4 times dispersion-centrifugation with a large excess of ethanol at room temperature. Then CNC-CA was dispersed in deionized water and dialysis 
against deionized water for one week. The CA dissolved in the washing ethanol were recycled by distillation of the solvent in a rotavapor. The content of carboxyl group in CNC-CA was conducted by potentiometric titration and the value is $0.679 \mathrm{mmol} / \mathrm{g}$, the test method is in accordance with our previous work (Zhou et al. 2018).

\section{Preparation of XSBR/CNC-CA nanocomposites}

The carboxyl content of XSBR was $2.59 \mathrm{mmol} / \mathrm{g}$, the specific test methods are presented in the supporting information. XSBR/CNC-CA nanocomposites films were prepared by a solution casting method. For the preparation of XSBR/CNC-CA nanocomposites with dual crosslinking networks, XSBR (3 $\mathrm{g}$ ) and the desired amount of CNC-CA, PEGDE, 2-methylimidazole were mixed together, ultrasonically treated for half an hour and then stirred for one hour. The obtained mixture was cast on a glass petri dish and dried by solvent evaporation at $60^{\circ} \mathrm{C}$ temperature for $12 \mathrm{~h}$, the samples were then further treated at $110^{\circ} \mathrm{C}$ for six hours to ensure complete crosslinking. The dual crosslinked XSBR/CNC-CA nanocomposites with $1 \%, 3 \%, 5 \%, 7 \%, 9 \%$ CNC-CA were prepared, the resulting sample is marked as $\mathrm{X} / \mathrm{mC}$ - $\mathrm{D}$ ( $\mathrm{m}$ represents the content of CNC-CA in nanocomposites, $\mathrm{X}, \mathrm{C}$, and $\mathrm{D}$ are the abbreviations of $X S B R, C N C-C A$ and dual crosslinking network, respectively). The mole of PEGDE is equal to the carboxyl content of CNC-CA in XSBR/CNC-CA nanocomposites. The mass ratio of 2-methylimidazole to PEGDE was 1:1. For comparison, the XSBR/CNC-CA nanocomposites without PEGDE and 2-methylimidazole were also prepared according to the above method, and the resulting sample is marked as $\mathrm{X} / \mathrm{mC}-\mathrm{H}(\mathrm{H}$ is the abbreviation of hydrogen bonded crosslinking network).

\section{Characterizations}

FTIR spectra were conducted on a Bruker tensor 70 spectrometer equipped with a DTG detector under the transmission mode in the wavenumber range 4000 to $400 \mathrm{~cm}^{-1}$ or under the ATR mode in the wavenumber range 4000 to $600 \mathrm{~cm}^{-1}$ with the resolution of $2 \mathrm{~cm}^{-1}$ and 32 scans.

The wide-angle X-ray diffraction (WAXD) patterns were recorded on a Rigaku mart Lab diffractometer with Cu Ka radiation $(\lambda=0.154 \mathrm{~nm})$ in the range $2 \theta=5-40^{\circ}$ with a scan rate of $5 \% \mathrm{~min}$.

Thermogravimetric analysis (TGA) measurements were carried out using a thermogravimetric analyzer (NETZSCH TG 209F1 Libra) under nitrogen gas flow at a rate of $100 \mathrm{~mL} / \mathrm{min}$. Samples were heated from 30 to $600^{\circ} \mathrm{C}$ at $10^{\circ} \mathrm{C} / \mathrm{min}$.

Dynamic light scattering (DLS) was used to characterize the average particle size of CNC-CA and CNC$\mathrm{OSO}_{3} \mathrm{H}$ in suspension. DLS was performed on a Malvern Nano ZS90 light scattering instrument.

The transmission electron microscopy (TEM) was conducted to observe the morphological differences of $\mathrm{CNC}-\mathrm{CA}$ and $\mathrm{CNC}-\mathrm{OSO}_{3} \mathrm{H}$. The aqueous solution of $\mathrm{CNC}-\mathrm{CA}$ and $\mathrm{CNC}-\mathrm{OSO}_{3} \mathrm{H}$ was treated by ultrasonic to obtain a uniform suspension. A small drop of $0.1 \mathrm{wt} \%$ solution was deposited on a carbon-coated copper grid. TEM observations were carried out on a JEM-2100 electron microscope operated at an acceleration voltage of $300 \mathrm{kV}$. 
SEM images were recorded with a Quanta FEG 250 scanning electron microscope. The cryogenically fractured surfaces of neat XSBR and XSBR/CNC-CA nanocomposites specimens were sputtered with gold before observations.

The tensile test was performed by Instron 5943 with a tensile rate of $50 \mathrm{~mm} / \mathrm{min}$. Before testing, neat XSBR and XSBR/CNC-CA nanocomposite films were cut into rectangle with dimensions of $30 \times 5 \times 0.2$ $\mathrm{mm}^{3}$. At least 5 specimens were measured for each sample.

The dynamic mechanical analyses (DMA) of neat XSBR and X/mC-D nanocomposites were determined with a TA Q800 at the oscillation frequency of $1.0 \mathrm{~Hz}$ and the heating rate of $3^{\circ} \mathrm{C} / \mathrm{min}$. The experiment was conducted in the tensile mode at temperature range of -100 to $100^{\circ} \mathrm{C}$.

The swelling behaviors in water and toluene were measured according to the following procedure: three preweighed $\left(\mathrm{m}_{0}\right)$ specimens of $\mathrm{X} / \mathrm{mC}$-D nanocomposites were immersed in deionized water or toluene at $60^{\circ} \mathrm{C}$ for $72 \mathrm{~h}$, respectively. Then the weights of the samples after swelling were recoded as $m_{1}$. The swelling ratios were calculated according to the following formula.

Swelling ratio $=\frac{m_{1}-m_{0}}{m_{0}} \times 100 \%$

The solubility of neat XSBR and XSBR/CNC-CA nanocomposites was conducted by immersing a certain weight of specimens into toluene/DMF mixed solution at $60^{\circ} \mathrm{C}$ for $72 \mathrm{~h}$, then ultrasound for an hour.

\section{Results And Discussion}

\section{Preparation and characterization of CNC-CA}

The preparation route and reaction mechanism of CA modified $\mathrm{CNC}-\mathrm{OSO}_{3} \mathrm{H}$ are presented in Scheme 1 . Large amount of hydroxyl group on the surface of $\mathrm{CNC}-\mathrm{OSO}_{3} \mathrm{H}$ reacted with carboxyl group of $\mathrm{CA}$ and sulfonate of $\mathrm{CNC}-\mathrm{OSO}_{3} \mathrm{H}$ was replaced by carboxyl group during the reaction. The FTIR spectra of CNC$\mathrm{CA}$ and $\mathrm{CNC}^{-} \mathrm{OSO}_{3} \mathrm{H}$ were provided in Fig. $1 \mathrm{a}$. It is worth noting that all CNC-CA were washed thoroughly to eliminate all residual reactants before analysis. As shown in Fig. 1a, in the FTIR spectra of CNC-CA, there is a new band appeared at $1725 \mathrm{~cm}^{-1}$ which corresponds to the $\mathrm{C}=0$ vibration from the ester

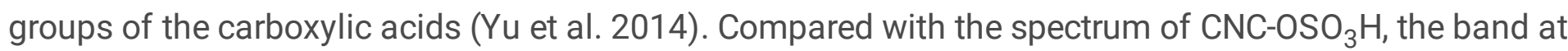
$812 \mathrm{~cm}^{-1}$ which corresponds to C-O-S vibration disappeared in CNC-CA (Wang et al. 2020). Those evidences suggest that the $\mathrm{CA}$ have been grafted successfully on $\mathrm{CNC}^{-} \mathrm{OSO}_{3} \mathrm{H}$ and most of the sulfonate on the surface of $\mathrm{CNC}-\mathrm{OSO}_{3} \mathrm{H}$ was removed during the modification process. The removal of sulfonate was also verified by EDS elemental analysis, as shown in table S1, after the surface modification, no sulfur element can be detected. 
The crystalline structure of $\mathrm{CNC}-\mathrm{CA}$ and $\mathrm{CNC}-\mathrm{OSO}_{3} \mathrm{H}$ were analyzed by X-ray diffractions. Both $\mathrm{CNC}-\mathrm{CA}$ and $\mathrm{CNC}-\mathrm{OSO}_{3} \mathrm{H}$ show diffraction peaks at $2 \theta$ of $14.8^{\circ}, 16.4^{\circ}$ and $22.7^{\circ}$, corresponding to the typical X-ray diffraction pattern of form-I crystal of cellulose. Based on the above investigation, it is confirmed that $\mathrm{CNC}-\mathrm{CA}$ has same crystalline structure as $\mathrm{CNC}^{-} \mathrm{OSO}_{3} \mathrm{H}$. The application of $\mathrm{CNC}-\mathrm{OSO}_{3} \mathrm{H}$ in polymer compounds is limited due to the poor heat resistance. Here the thermal degradation of CNC-CA and CNC$\mathrm{OSO}_{3} \mathrm{H}$ was compared by thermogravimetric analysis (TGA). As shown in Fig. $1 \mathrm{c}$. and d, CNC-CA displays a markedly enhanced thermal stability compared with $\mathrm{CNC}^{-} \mathrm{OSO}_{3} \mathrm{H}$. The maximum weight-loss rate temperature of $\mathrm{CNC}-\mathrm{CA}$ is $354^{\circ} \mathrm{C}$, which is $143^{\circ} \mathrm{C}$ higher than that of $\mathrm{CNC}-\mathrm{OSO}_{3} \mathrm{H}$. According to the FTIR results of the $\mathrm{CNC}-\mathrm{CA}$ and $\mathrm{CNC}-\mathrm{OSO}_{3} \mathrm{H}$, it is deduced that removal of the sulfate groups is a crucial factor in improving the thermal stability of CNCs.

The morphology of $\mathrm{CNC}-\mathrm{OSO}_{3} \mathrm{H}$ and $\mathrm{CNC}-\mathrm{CA}$ were observed by using TEM (shown in Fig. 2a and b). The individual cellulose nanocrystals of both $\mathrm{CNC}-\mathrm{OSO}_{3} \mathrm{H}$ and $\mathrm{CNC}-\mathrm{CA}$ can be clearly distinguished.

Compared with $\mathrm{CNC}-\mathrm{OSO}_{3} \mathrm{H}$, the surface of $\mathrm{CNC}-\mathrm{CA}$ is much more blurry because the surface of $\mathrm{CNC}-\mathrm{CA}$ is covered with small molecules of citric acid. Because of the strong hydrogen bond interaction between the carboxyl groups of citric acid, CNC-CA is more likely to agglomerate after drying. The average particle sizes of $\mathrm{CNC}^{-} \mathrm{OSO}_{3} \mathrm{H}$ and $\mathrm{CNC}-\mathrm{CA}$ were estimated by dynamic mechanical analyses (DLS), the values are around $187 \mathrm{~nm}$ and $161 \mathrm{~nm}$, respectively. The decrease of the size of cellulose nanocrystals after modification may be due to the further etching of the amorphous region on the surface of $\mathrm{CNC}-\mathrm{OSO}_{3} \mathrm{H}$ by CA.

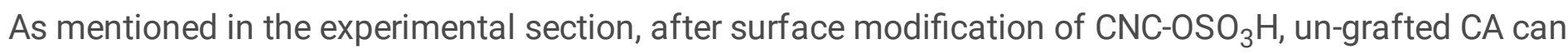
be separated and reused. To examine the effectiveness of recycled CA, CNC-CA are prepared by using pristine CA, first and second recycled CA as the modifiers. The results are shown in Fig. S1a and b. It can be observed that all these three samples can be stably dispersed in deionized water. In addition, the similar FTIR spectra of these three kinds of CNC-CA indicates that recycled CA can also modify CNC$\mathrm{OSO}_{3} \mathrm{H}$ effectively.

\section{Mechanical Properties of XSBR/CNC-CA Nanocomposites}

In order to study the effect of dual crosslinking network on the mechanical properties of XSBR nanocomposites, the tensile tests were performed. The tensile curves and mechanical property parameters of neat XSBR, X/mC-H and X/mC-D nanocomposites containing 1, 3, 5, 7 and $9 \mathrm{wt} \% \mathrm{CNC}$-CA are shown in Fig. 3. For neat XSBR, the stress increased slowly until break, and achieved tensile strength and strain at failure of $2.56 \mathrm{MPa}$ and $389 \%$, respectively. For the $\mathrm{X} / \mathrm{mC}-\mathrm{H}$ nanocomposites, even though there are some improvement by adding CNC-CA into the XSBR matrix, the improvement is quite limited (shown in Fig. 3a). XSBR is not crosslinked by covalent bonds in the $\mathrm{X} / \mathrm{mC}-\mathrm{H}$ nanocomposites, and the role of hydrogen bond is very limited. The tensile strength of $\mathrm{X} / \mathrm{mC}-\mathrm{H}$ nanocomposites is far lower than the requirement of industrial application. Therefore, covalent crosslinking of the nanocomposites is an essential step to further improve its properties. Here, end-epoxy group PEGDE was used as crosslinking 
agent to realize chemical crosslinking of nanocomposites through the reaction between epoxy group of PEGDE and carboxyl group of XSBR and CNC-CA. As expected, the mechanical properties of X/mC-D nanocomposites show a significant improvement compared to the $\mathrm{X} / \mathrm{mC}-\mathrm{H}$ ones (as shown in Fig. $3 \mathrm{~b}$ ). For example, with only $5 \mathrm{wt} \% \mathrm{CNC}-\mathrm{CA}$ addition, tensile strength and strain at failure of X/5C-D nanocomposites increase to $8.59 \mathrm{MPa}$ and $456 \%$, respectively, which are even superior to that of $\mathrm{X} / 9 \mathrm{C}-\mathrm{H}$ nanocomposites. When the content of CNC-CA increases to $9 \mathrm{wt} \%$, the X/9C-D nanocomposites show tensile strength of $12.65 \mathrm{MPa}$, which are nearly five times that of the neat XSBR, and it's valuable that X/9C-D keeps same elongation at break with pure XSBR. Moreover, from the charts of Fig. 3c. and d., it can be seen that the tensile stresses of the nanocomposites with dual crosslinking networks are nearly double of that with only hydrogen bond, moreover, the fracture energy of the samples with dual crosslinking networks is also significantly improved.

\section{Dynamic mechanical performance of XSBR/CNC-CA Nanocomposites}

The temperature dependence of the storage modulus ( $\left.E^{\prime}\right)$ and the loss tangent (tan $\left.\delta\right)$ for neat XSBR and $\mathrm{X} / \mathrm{mC}$-D nanocomposites with different CNC-CA contents are shown in Fig. 4a and Fig. 4b. A successive increase of the $E^{\prime}$ values can be observed by increasing the amount of CNC-CA (as shown in Fig. 4a.). For example, the storage modulus at $-70^{\circ} \mathrm{C}$ increases from 1049 to $3083 \mathrm{MPa}$ with the addition of CNC-CA from 0 to $9 \mathrm{wt} \%$ in the XSBR matrix. The enhancement in modulus below glass transition temperature is a good evidence for the strong reinforced tendency of CNC-CA in the XSBR matrix. Meanwhile, the improvement of crosslinking degree brought by the dual crosslinking network (the covalent bond networks and the $\mathrm{H}$-bond networks) also contributes to the improvement of the modulus.

Figure 4b. shows the variation of tand as a function of temperature for the neat XSBR and X/mC-D nanocomposites. XSBR exhibits a peak tan $\delta$ value at a temperature around $-3^{\circ} \mathrm{C}$, which corresponds to the glass transition temperature $\left(T_{g}\right)$ of XSBR. While the $T_{g}$ of $X / m C-D$ nanocomposites gradually decreases to lower temperature (to $-6^{\circ} \mathrm{C}$ ) with the increase of CNC-CA content. Two effects might decrease the $\mathrm{T}_{\mathrm{g}}$ value: one is, as reported by Cao et. al, the hydrogen bond formed between filler particles and matrix may act as an internal plasticization resulting in the shifting of $T_{g}$ to a lower temperature with increasing filler content (Cao et al. 2013); another is, excessive 2-methylimidazole might act as plasticizer in the $\mathrm{X} / \mathrm{mC}$-D nanocomposites because the amount of 2-methylimidazole used is proportional to the CNC-CA content in the nanocomposite. However, further research is needed to clarify this question. Besides, the intensity of $\tan \delta$, which indicates the damping properties, decreases with the increase of CNC-CA content, which is mainly due to the decrease of matrix content and the enhancement of filler's restriction effect on the chain mobility of rubber matrix.

\section{The dispersion of CNC-CA in XSBR/CNC-CA Nanocomposites}


The uniform dispersion of fillers in the matrix plays a key role on improving the properties of nanocomposites. The aggregation of fillers in the matrix forms stress concentration points and has adverse effects on the properties of the nanocomposites.

Figure 5a-f. illustrate the SEM images of cryogenically fractured surfaces of the neat XSBR and X/mC-D nanocomposites containing 1, 3, 5, 7 and 9 wt\% CNC-CA, respectively. Compared to the micrograph of neat XSBR, the morphology of CNC-CA in the XSBR matrix can be easily identified. The CNC-CA appears as white dots and their concentration on the fracture surface of the nanocomposites increases directly with the increase of the CNC-CA loading. A uniform distribution of CNC-CA in the XSBR matrix can be observed in all X/mC-D nanocomposites (as shown in Fig. 5b-f). It is obvious that those CNC-CA dots are partial embedded in XSBR matrix, demonstrating good compatibility between the fillers and matrix. Furthermore, no gaps, voids or pull-out cracks are observed on the surface, indicating excellent interfacial adhesion between the CNC-CA and XSBR matrix. This excellent compatibility and interface adhesion can be ascribed to the hydrogen bond between XSBR and CNC-CA and the formation of ester group among PEGDE, XSBR and CNC-CA. This uniform distribution and excellent interfacial adhesion are anticipated to play an important role on the improvement of the mechanical performance of the resulting $\mathrm{X} / \mathrm{mC}-\mathrm{D}$ nanocomposites.

\section{Swelling properties and solubility of XSBR/CNC-CA Nanocomposites}

The water and toluene uptake ratios of X/mC-D nanocomposites containing 1, 3, 5, 7 and 9 wt\% CNC-CA were conducted to investigate the hydrophilicity and crosslinking degree. The $\mathrm{X} / \mathrm{mC}$ - $\mathrm{D}$ nanocomposites were immersed in deionized water or toluene for $72 \mathrm{~h}$ at $60^{\circ} \mathrm{C}$ and weighed. Figure 6 . shows the water uptake ratios of neat XSBR and X/mC-D nanocomposites. The water uptake ratios of X/mC-D nanocomposites with CNC-CA content $\leq 7 \mathrm{wt} \%$ was much lower than that of neat XSBR. This may be attributed to the dual crosslinking network which makes the components of nanocomposites compact more densely and restricts the chain mobility of the rubber matrix, subsequently inhibits the diffusion of water in the nanocomposites effectively. But, the hydrophilicity of $\mathrm{X} / \mathrm{mC}$-D nanocomposites increases with the increase of hydrophilic CNC-CA content, especially when the CNC-CA content $>7 \mathrm{wt} \%$, there is a dramatic increase of water uptake ratios with increase of CNC-CA content. As can be seen from Fig. 6 , the water uptake ratios of X/9C-D is almost twice that of neat XSBR. This phenomenon is due to the dual role of CNC-CA in X/mC-D nanocomposites. On one hand, the formation of hydrogen bond between CNC-CA and XSBR molecular chains inhibits the movement of XSBR molecular chains and reduces the possibility of hydrogen bond formation between XSBR molecular chains and water, resulting in the decrease of hydrophilicity of $\mathrm{X} / \mathrm{mC}$-D nanocomposites. But, due to hydrogen bonds have saturability, when CNC-CA content reaches a certain level, the number of hydrogen bond between CNC-CA and XSBR molecular chains remains unchanged with increase of CNC-CA. So, the effect of hydrogen bond on the hydrophilicity of X/mC-D nanocomposites doesn't increase when CNC-CA content reaches a certain level. On the other hand, CNC-CA has the inherent hydrophilicity, more CNC-CA, more hydrophilicity is introduced in the $\mathrm{X} / \mathrm{mC}$-D nanocomposites. As a result of the above two aspects, water uptake ratios of $\mathrm{X} / \mathrm{mC}-\mathrm{D}$ 
nanocomposites increase with increase of CNC-CA content and has an interrupt increase when the content of CNC-CA $>7 \%$. On the contrary, the toluene absorption ratios decrease with the increasement of CNC-CA content, which should be attributed to the increased hydrophilicity caused by the introduction of CNC-CA and the dual crosslinking network restricts the chain mobility of XSBR.

In order to confirm the formation of dual crosslinking network, the $\mathrm{X} / \mathrm{mC}-\mathrm{D}$ and $\mathrm{X} / \mathrm{mC}-\mathrm{H}$ nanocomposites were immersed in DMF/toluene mixed solution for $72 \mathrm{~h}$ at $60^{\circ} \mathrm{C}$ and ultrasound treating for $1 \mathrm{~h}$ to check the swelling and dissolution behavior. As shown in Fig. S2, all the $\mathrm{X} / \mathrm{mC}-\mathrm{H}$ nanocomposites sheets (upper) and neat XSBR disintegrated and dissolved in the DMF/toluene mixed solvent. In sharp contrast, the shape of all the X/mC-D nanocomposites sheets (below) keep well. The above experimental phenomena further prove that PEGDE has an effective crosslinking effect on the XSBR/CNC-CA nanocomposite.

\section{Structural Characterization and proposed dual crosslinking networks of XSBR/CNC-CA nanocomposites Structural Characterization of XSBR/CNC-CA nanocomposites}

FTIR is a powerful tool for studying the intermolecular interactions, hydrogen bonds and chemical reactions. The FTIR spectra of neat XSBR, PEGDE, XSBR/PEGDE, CNC-CA, and CNC-CA/PEGDE are shown in Fig. 7. In order to make the results clear, here, the mass ratios of XSBR to PEGDE and CNC-CA to PEGDE were set to be $1: 1$, and the samples were treated at $110^{\circ} \mathrm{C}$ for six hours. As shown in Fig. 7, the three peaks of $3055,1252,854 \mathrm{~cm}^{-1}$ in the FTIR spectrum of PEGDE are the characteristic peaks of epoxy group. Compared to the FTIR spectra of single XSBR and PEGDE, a new band at $1725 \mathrm{~cm}^{-1}$, which corresponds to the $\mathrm{C}=0$ vibrations of ester group, appeared in the XSBR/PEGDE blends as shown in Fig. $7 a$. This reveals that the carboxyl groups of XSBR reacted with the epoxy groups of PEGDE and formed $\beta$ hydroxyl esters. The spectra of CNC-CA/PEGDE blends, is shown in Fig. 7b. The three characteristic peaks of PEGDE disappeared in the FTIR spectrum of CNC-CA/PEGDE blends, which means that the carboxyl group of CNC-CA and the epoxy group of PEGDE are esterified. From the above analysis results, we confirmed that the epoxy group on PEGDE can react with carboxyl group of both XSBR and CNC-CA, therefore, PEGDE can effectively crosslink XSBR/CNC-CA nanocomposites.

The existence of hydrogen bonds in X/mC-D nanocomposites was also confirmed by FTIR. As shown in Fig. S3, the intensity of the band between $3500-3250 \mathrm{~cm}^{-1}$ corresponding to the hydrogen bond became stronger after compounding the CNC-CA with XSBR, indicating there is a strong hydrogen bond interaction between XSBR and CNC-CA. According to the above results, it can be concluded that dual crosslinking networks of the covalent bond networks and the hydrogen bond networks have been formed in XSBR/CNC-CA nanocomposites, both contribute to improve the mechanical properties of XSBR/CNCCA nanocomposites. 


\section{The proposed model of dual crosslinking networks for crosslinked XSBR/CNC-CA nanocomposites}

Based on the above discussion, it can be concluded that dual crosslinking networks featured of hydrogen bonds network and covalent bonds network have been formed in X/mC-D nanocomposite. The esterification of epoxy group of PEGDE with carboxyl group of XSBR and CNC-CA is shown in Scheme S1, the illustration of hydrogen bonds network and dual crosslinking network is shown in Scheme 2.

Compared with the ionic bond and covalent bond, hydrogen bond is much weaker so that its ability on the improvement of the mechanical properties of $\mathrm{XSBR} / \mathrm{mC}-\mathrm{H}$ is very limited. By further constructing covalent bonds in the $\mathrm{X} / \mathrm{mC}$-D nanocomposites using PEGDE as crosslinking agent, the rubber chains and CNC-CA are held together tightly. The combination of the two networks can effectively improve the compatibility and interface adhesion between CNC-CA and XSBR, as well as the interactions between XSBR-XSBR, which is beneficial to the improvement of the physical and mechanical properties of XSBR/CNC-CA nanocomposites.

\section{Conclusions}

In this work, a green and environment friendly method of $\mathrm{CA}$ grafting $\mathrm{CNC}-\mathrm{OSO}_{3} \mathrm{H}$ was provided. Asprepared CNC-CA has higher thermal stability and excellent compatibility with XSBR. Meanwhile the dual crosslinking networks in XSBR/CNC-CA nanocomposites were constructed. By using PEGDE as the crosslinker, covalent bonds which serves as the chemical crosslinking network were formed between the XSBR and CNC-CA and PEGDE. The hydrogen bond formed between XSBR and CNC-CA serves as the physical crosslinking network. Besides, 2-methylimidazole the catalyst for esterification acts as plasticizer to endow the XSBR/CNC-CA nanocomposites with better ductility. The combination of dual crosslinking networks and excessive 2-methylimidazole is beneficial for the improvement of the strength and ductility of XSBR/CNC-CA nanocomposites. As CNC-CA content reaches to $9 \mathrm{wt} \%$, the tensile strength of the $\mathrm{X} / \mathrm{mC}$-D nanocomposite is five times that of the neat XSBR with the same elongation at break. Beyond that, the addition of CNC-CA improves the solvent resistance of XSBR nanocomposites. This research provides a facile way to the surface modification of $\mathrm{CNC}^{-} \mathrm{OSO}_{3} \mathrm{H}$ and the fabrication of dual crosslinking XSBR nanocomposites with superior properties.

\section{Declarations}

\section{Supporting Information Available}

Element analysis data of $\mathrm{CNC}^{-} \mathrm{OSO}_{3} \mathrm{H}$ and $\mathrm{CNC}-\mathrm{CA}$; Photograph and FTIR spectra of CNC-CA-1st, CNC-CA2nd and CNC-CA-3rd; The dissolution and swelling pictures of neat XSBR, X/mC-H and X/mC-D nanocomposites in DMF/toluene solution; FTIR spectra of neat XSBR, CNC-CA and X/mC-D 
nanocomposites; The esterification involved in the preparation of $\mathrm{X} / \mathrm{mC}$-D nanocomposites; The determination of carboxyl content in XSBR.

\section{Acknowledgements}

The authors acknowledge the financial support from National Natural Science Foundation of China (21774068 and 51903135).

Ethical approval Not applicable

Ethical standards Compliance with ethical standards

Conflict of interest The authors declare no competing financial interest.

\section{References}

1. Bitinis N, Hernandez M, Verdejo R, Kenny JM, Lopez-Manchado MA. (2011). Recent advances in clay/polymer nanocomposites. Advanced Materials, 23, 5229-5236. https://doi.org/10.1002/adma.201101948

2. Blanchard R, Ogunsona EO, Hojabr S, Berry R, Mekonnen TH. (2020). Synergistic Cross-linking and Reinforcing Enhancement of Rubber Latex with Cellulose Nanocrystals for Glove Applications. ACS Applied Polymer Materials, 2, 887-898. https://doi.org/10.1021/acsapm.9b01117

3. Cao L, Huang J, Chen Y. (2018). Dual Cross-linked Epoxidized Natural Rubber Reinforced by Tunicate Cellulose Nanocrystals with Improved Strength and Extensibility. ACS Sustainable Chemistry \& Engineering, 6, 14802-14811. https://doi.org/10.1021/acssuschemeng.8b03331

4. Cao X, Xu C, Liu Y, Chen Y. (2013). Preparation and properties of carboxylated styrene-butadiene rubber/cellulose nanocrystals composites. Carbohydrate Polymers, 92, 69-76. https://doi.org/10.1016/j.carbpol.2012.09.054

5. Chen C, Justice RS, Schaefer DW, Baur JW. (2008). Highly dispersed nanosilica-epoxy resins with enhanced mechanical properties. Polymer, 49, 3805-3815 https://doi.org/10.1016/j.polymer.2008.06.023

6. Chen Y, Xu C, Cao X. (2015). Dynamic rheology studies of carboxylated butadiene-styrene rubber/cellulose nanocrystals nanocomposites: Vulcanization process and network structures. Polymer Composites, 36, 623-629. https://doi.org/10.1002/pc.22979

7. Ding B, Huang S, Shen K, Hou J, Gao H, Duan Y, Zhang J. (2019). Natural rubber bio-nanocomposites reinforced with self-assembled chitin nanofibers from aqueous $\mathrm{KOH} /$ urea solution. Carbohydrate Polymers, 225, 115-230. https://doi.org/10.1016/j.carbpol.2019.115230

8. Dufresne A. (2018). Cellulose nanomaterials as green nanoreinforcements for polymer nanocomposites. Philosophical transactions of the royal society A, 376 (2112) https://doi.org/10.1098/rsta.2017.0040 
9. Eslami H, Tzoganakis C, Mekonnen TH. (2020). Surface graft polymerization of lactic acid from the surface of cellulose nanocrystals and applications in chloroprene rubber film composites. Cellulose, 27, 5267-5284. https://doi.org/10.1007/s10570-020-03167-w

10. Flauzino Neto WP, Mariano M, Dufresne A. (2016). Mechanical properties of natural rubber nanocomposites reinforced with high aspect ratio cellulose nanocrystals isolated from soy hulls. Carbohydrate Polymers, 153,143-152 https://doi.org/10.1016/j.carbpol.2016.07.073

11. Favier V, Chanzy H, Cavaille JY. (1995). Polymer Nanocomposites Reinforced by Cellulose Whiskers. Macromolecules, 28, 6365-6367. https://doi.org/10.1021/ma00122a053

12. Habibi Y, L A. Lucia, O J. Rojas. (2010). Cellulose Nanocrystals: Chemistry, Self Assembly, and Applications. Chemical Reviews, 110, 3479-3500. https://doi.org/10.1021/cr900339w

13. Iwatake A, Nogi M, Yano H. (2008). Cellulose nanofiber-reinforced polylactic acid. Composites Science and Technology, 68, 2103-2106. https://doi.org/10.1016/j.compscitech.2008.03.006

14. Ji H, Xiang Z, Qi H, Han T, Pranovich A, Song T. (2019). Strategy towards one-step preparation of carboxylic cellulose nanocrystals and nanofibrils with high yield, carboxylation and highly stable dispersibility using innocuous citric acid. Green Chemistry, 21, 1956-1964. https://doi.org/10.1039/c8gc03493a

15. Jiang F, Esker AR, Roman M. (2010). Acid-catalyzed and solvolytic desulfation of $\mathrm{H}_{2} \mathrm{SO}_{4}$-hydrolyzed cellulose nanocrystals. Langmuir, 26, 17919-17925. https://doi.org/10.1021/la1028405

16. Jiang W, Gu J. (2020). Nanocrystalline cellulose isolated from different renewable sources to fabricate natural rubber composites with outstanding mechanical properties. Cellulose, 27, 58015813. https://doi.org/10.1007/s10570-020-03209-3

17. Kargarzadeh H, Huang J, Lin N, Ahmad I, Gałęski A. (2018). Recent developments in nanocellulosebased biodegradable polymers, thermoplastic polymers, and porous nanocomposites. Progress in Polymer Science, 87, 197-227. https://doi.org/10.1016/j.progpolymsci.2018.07.008

18. Kargarzadeh H, Mariano M, Huang J, Lin N, Ahmad I, Dufresne A, Thomas S. (2017). Recent developments on nanocellulose reinforced polymer nanocomposites: A review. Polymer, 132, 368393. https://doi.org/10.1016/j.polymer.2017.09.043

19. Klemm D, Cranston E.D, Kralisch D. (2018). Nanocellulose as a natural source for groundbreaking applications in materials science: Today's state. Materials Today, 21, 720-748. https://doi.org/10.1016/j.mattod.2018.02.001

20. Nunes RCR. (2017). Rubber nanocomposites with nanocellulose. Progress in Rubber Nanocomposites, 13, 463-494. https://doi.org/10.1016/b978-0-08-100409-8.00013-9

21. Parambath Kanoth B, Claudino M, Johansson M, Berglund LA, Zhou Q. (2015). Biocomposites from Natural Rubber: Synergistic Effects of Functionalized Cellulose Nanocrystals as Both Reinforcing and Cross-Linking Agents via Free-Radical Thiol-ene Chemistry. ACS Applied Materials \& Interfaces, 7, 16303-16310. https://doi.org/10.1021/acsami.5b03115

22. Peng C, Dong B, Zhang C, Hu Y, Liu L, Zhang X. (2018). A Host-Guest Interaction Assisted Approach for Fabrication of Polybutadiene Nanocomposites Reinforced with Well-Dispersed Cellulose 
Nanocrystals. Macromolecules, 51, 4578-4587. https://doi.org/10.1021/acs.macromol.8b00606

23. Peng C, Yang Q, Zhao W, Ren J, Yu Q, Hu Y, Zhang X. (2019). Relationship between interface chemistry and reinforcement in polybutadiene/cellulose nanocrystal nanocomposites. Composites Science and Technology, 177, 103-110. https://doi.org/10.1016/j.compscitech.2019.04.029

24. Potts JR, Shankar O, Du L, Ruoff RS. (2012). Processing-Morphology-Property Relationships and Composite Theory Analysis of Reduced Graphene Oxide/Natural Rubber Nanocomposites. Macromolecules, 45, 6045-6055. https://doi.org/10.1021/ma300706k

25. Ramorino G, Bignotti F, Pandini S, Riccò T. (2009). Mechanical reinforcement in natural rubber/organoclay nanocomposites. Composites Science and Technology, 69, 1206-1211. https://doi.org/10.1016/j.compscitech.2009.02.023

26. Roman M, Winter WT. (2004). Effect of Sulfate Groups from Sulfuric Acid Hydrolysis on the Thermal Degradation Behavior of Bacterial Cellulose. Biomacromolecules, 5, 1671-1677. https://doi.org/10.1021/bm034519+

27. Rooj S, Das A, Thakur V, Mahaling RN, Bhowmick AK, Heinrich G. (2010). Preparation and properties of natural nanocomposites based on natural rubber and naturally occurring halloysite nanotubes. Materials \& Design, 31, 2151-2156. https://doi.org/10.1016/j.matdes.2009.11.009

28. Sengupta R, Bhattacharya M, Bandyopadhyay S, Bhowmick AK. (2011). A review on the mechanical and electrical properties of graphite and modified graphite reinforced polymer composites. Progress in Polymer Science, 36, 638-670. https://doi.org/10.1016/j.progpolymsci.2010.11.003

29. Tang Z, Wu X, Guo B, Zhang L, Jia D. (2012). Preparation of butadiene-styrene-vinyl pyridine rubbergraphene oxide hybrids through co-coagulation process and in situ interface tailoring. Journal of Materials Chemistry, 22, 7492-7501 https://doi.org/10.1039/c2jm00084a

30. Tao H, Dufresne A, Lin N. (2019). Double-Network Formation and Mechanical Enhancement of Reducing End-Modified Cellulose Nanocrystals to the Thermoplastic Elastomer Based on Click Reaction and Bulk Cross-Linking. Macromolecules, 52, 5894-5906. https://doi.org/10.1021/acs.macromol.9b01213

31. Thomas B, Sanchez C. (2018). Nanocellulose, a Versatile Green Platform: From Biosources to Materials and Their Applications. Chemical Reviews, 118, 11575-11625. https://doi.org/10.1021/acs.chemrev.7b00627

32. Tian M, Zhen X, Wang Z, Zou H, Zhang L, Ning N. (2017). Bioderived Rubber-Cellulose Nanocrystal Composites with Tunable Water-Responsive Adaptive Mechanical Behavior. ACS Applied Materials \& Interfaces, 9, 6482-6487. https://doi.org/10.1021/acsami.6b16308

33. Wang L, Hu J, Liu Y, Shu J, Wu H, Zhang J. (2020). lonic Liquids Grafted Cellulose Nanocrystals for High-Strength and Toughness PVA Nanocomposite. ACS Applied Materials \& Interfaces, 12, 3879638804. https://doi.org/10.1021/acsami.0c11217

34. Wang N, Ding E, Cheng R. (2007). Thermal degradation behaviors of spherical cellulose nanocrystals with sulfate groups. Polymer, 48, 3486-3493 https://doi.org/10.1016/j.polymer.2007.03.062 
35. Wu H, Nagarajan S, Shu J, Zhang T, Zhou L, Duan Y, Zhang J. (2018). Green and facile surface modification of cellulose nanocrystal as the route to produce poly(lactic acid) nanocomposites with improved properties. Carbohydrate Polymers, 197, 204-214.

https://doi.org/10.1016/j.carbpol.2018.05.087

36. Yu H-Y, Chen G-Y, Wang Y-B, Yao J-M. (2014). A facile one-pot route for preparing cellulose nanocrystal/zinc oxide nanohybrids with high antibacterial and photocatalytic activity. Cellulose, 22, 261-273. https://doi.org/10.1007/s10570-014-0491-0

37. Yu H-Y, Zhang D-Z, Lu F-F, Yao J. (2016). New Approach for Single-Step Extraction of Carboxylated Cellulose Nanocrystals for Their Use As Adsorbents and Flocculants. ACS Sustainable Chemistry \& Engineering, 4, 2632-2643. https://doi.org/10.1021/acssuschemeng.6b00126

38. Zhou L, Li N, Shu J, Liu Y, Wang K, Zhang J. (2018). One-Pot Preparation of Carboxylated Cellulose Nanocrystals and Their Liquid Crystalline Behaviors. ACS Sustainable Chemistry \& Engineering, 6 , 12403-12410. https://doi.org/10.1021/acssuschemeng.8b02926

\section{Figures}
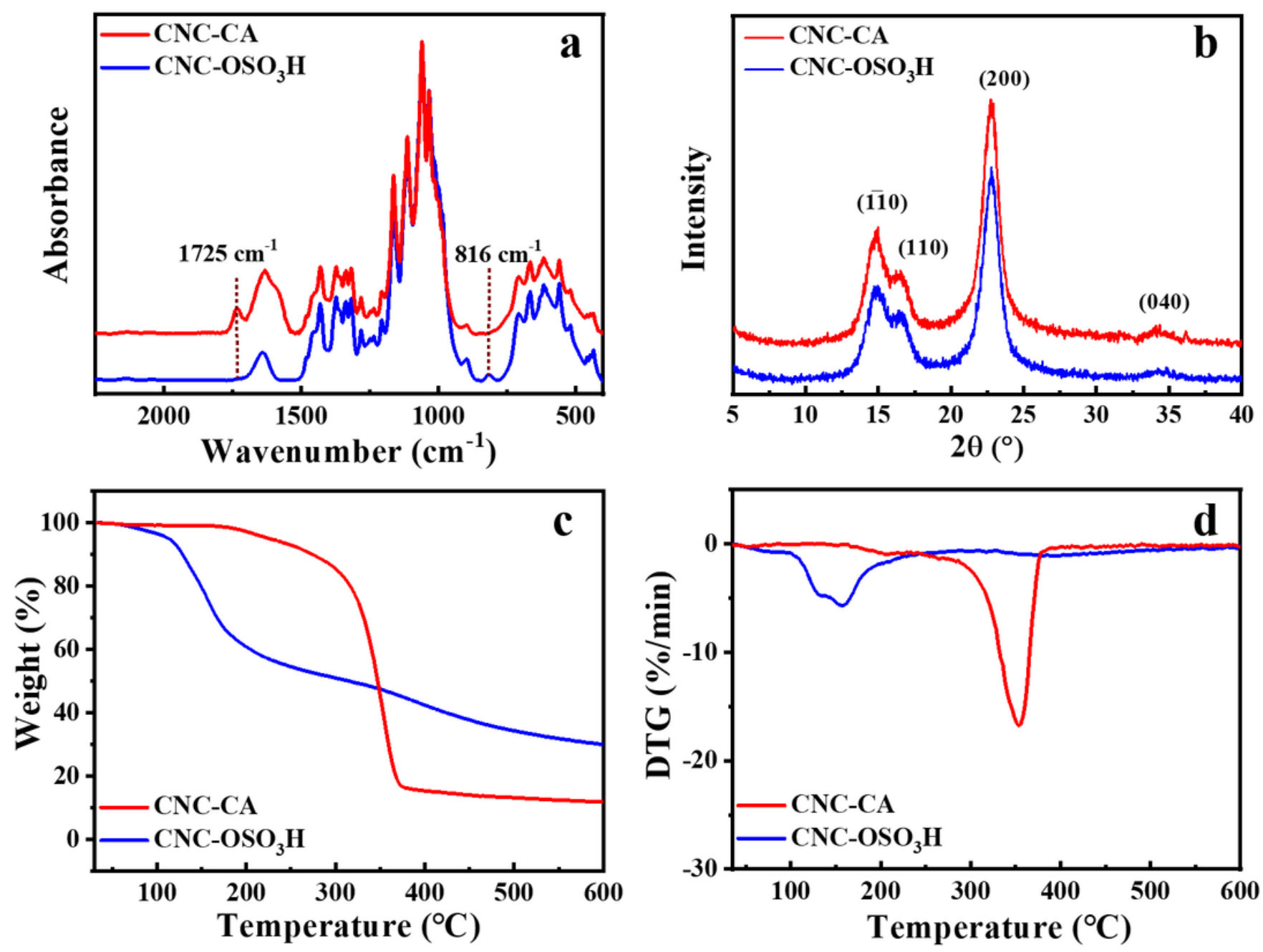
Figure 1

(a) FTIR spectra, (b) XRD patterns of CNC-CA and CNC-OSO3H, (c) TGA thermograms, (d) Derivative thermogravimetry of CNC-CA and CNC-OSO3H.
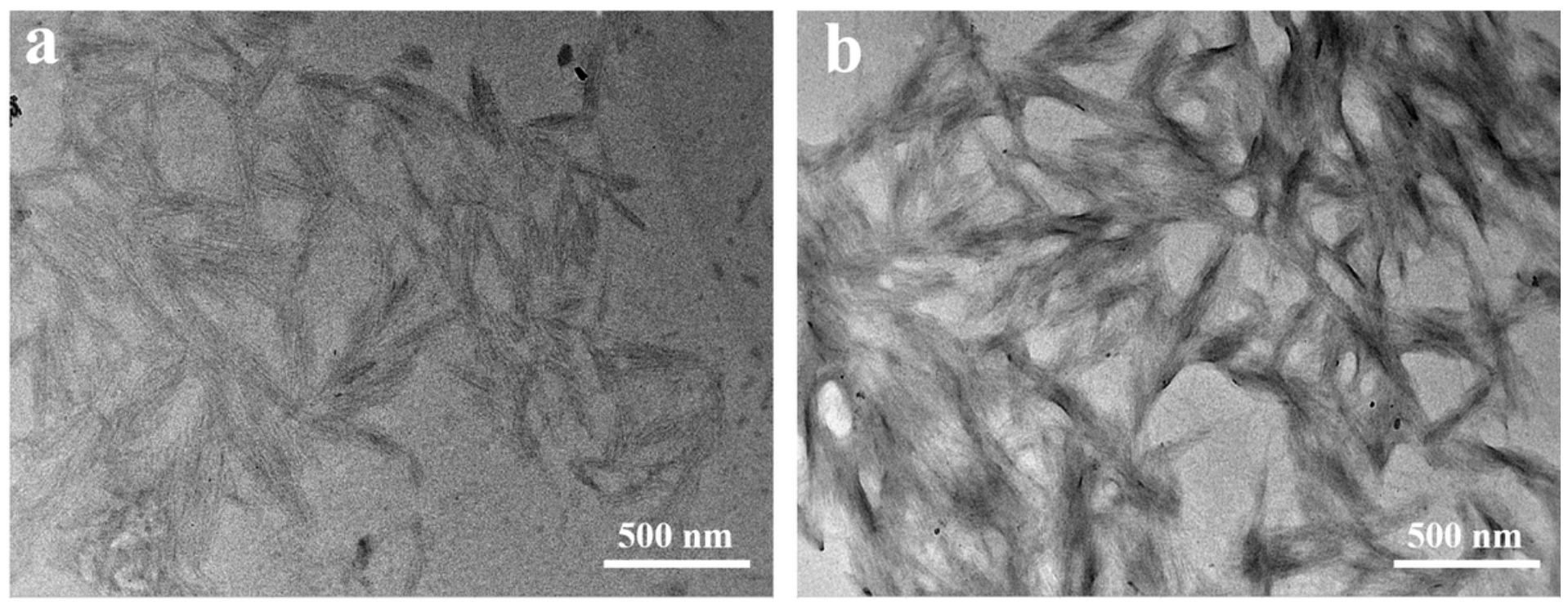

Figure 2

TEM micrographs of (a) CNC-OSO3H and (b) CNC-CA. 

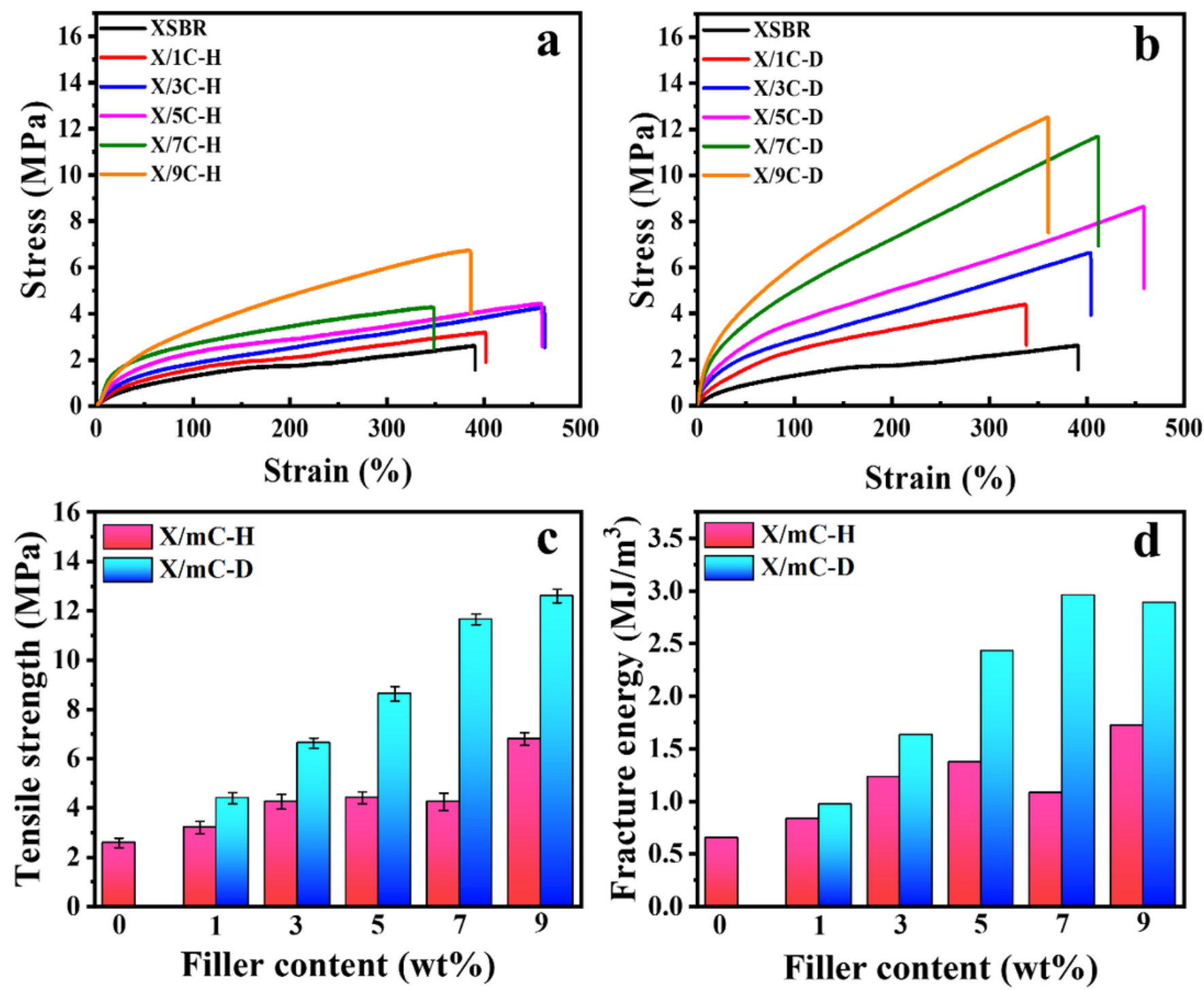

Figure 3

The tensile stress-strain curves of (a) neat XSBR and X/mC-H nanocomposites, (b) neat XSBR and X/mC$\mathrm{D}$ nanocomposites, (c) the tensile strength and (d) fracture energy as a function of filler contents. 

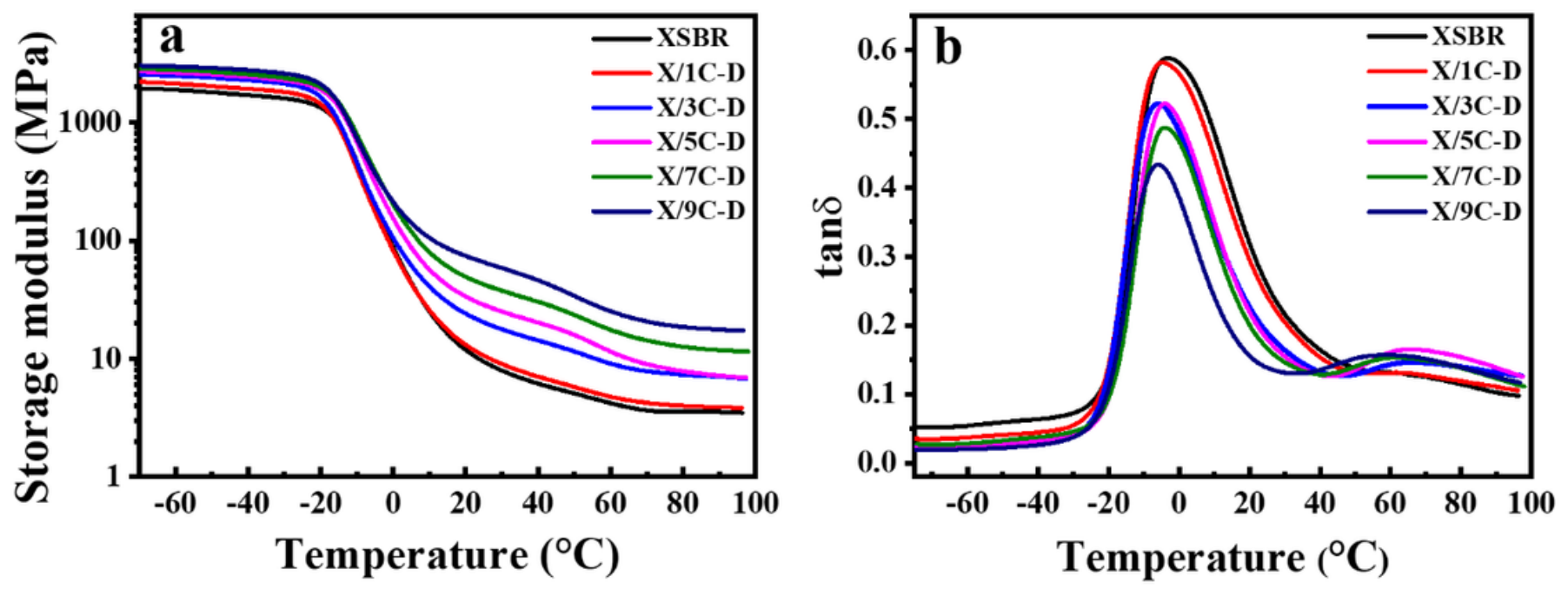

Figure 4

The temperature dependence of the storage modulus $\left(E^{\prime}\right)(a)$ and the loss tangent $(\tan \delta)$ (b) for neat XSBR and X/mC-D nanocomposites.
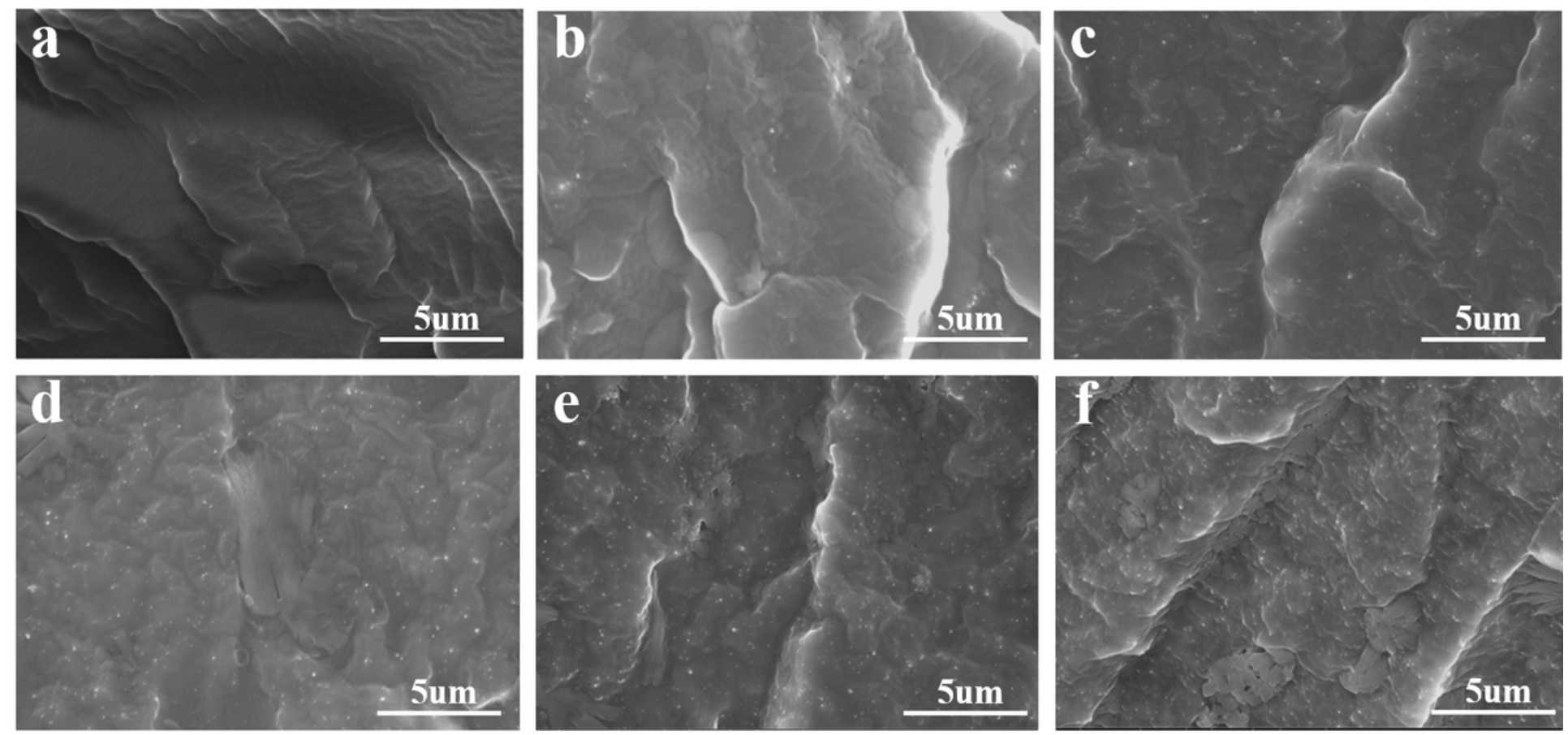

Figure 5

SEM images of the cryogenically fractured surfaces of neat XSBR and X/mC-D nanocomposites: (a) XSBR, (b)X/1C-D, (c) X/3C-D, (d) X/5C-D, (e) X/7C- D, (f) X/9C-D. 


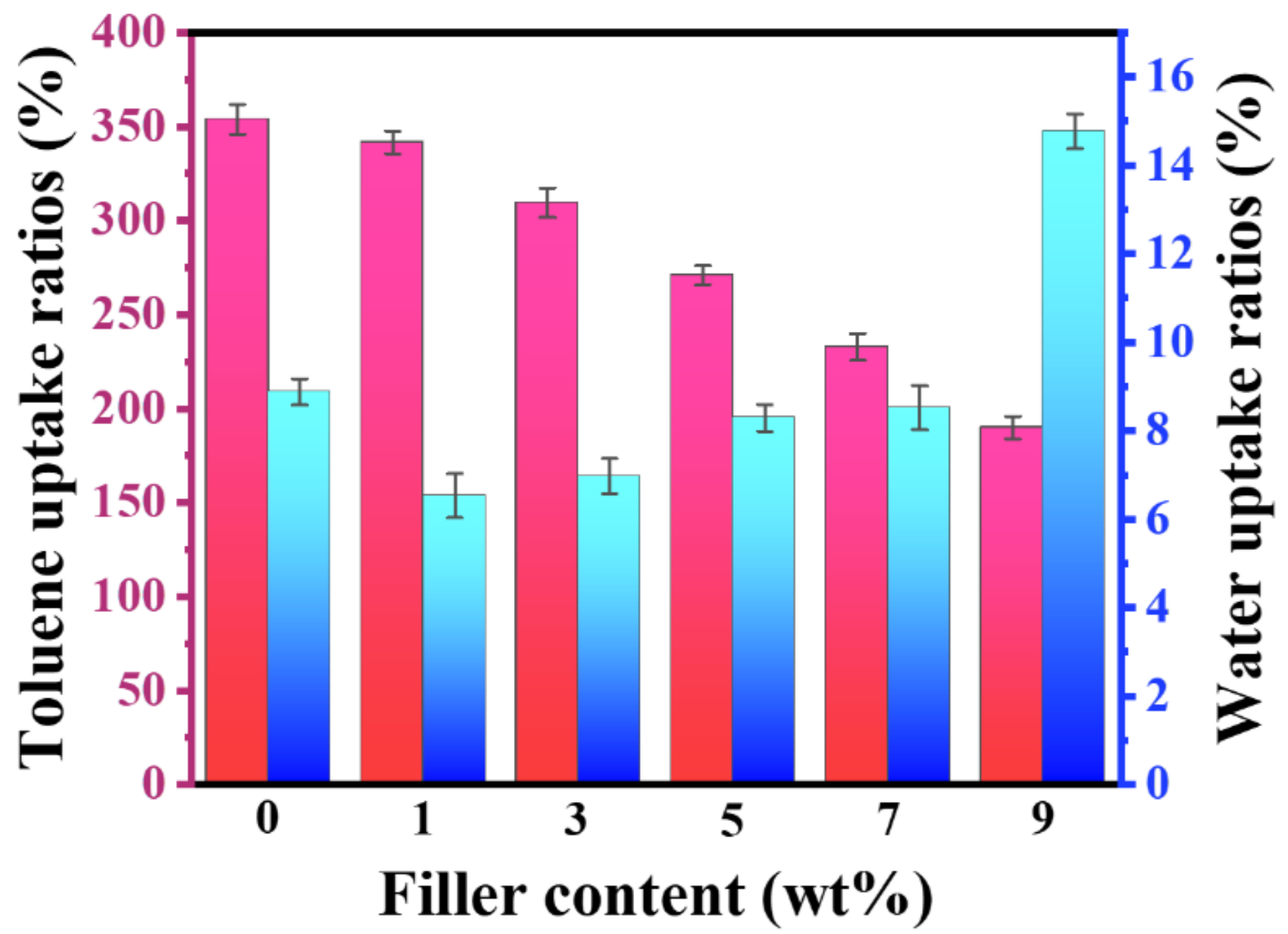

Figure 6

Toluene and water uptake ratios of neat XSBR and X/mC-D nanocomposites.
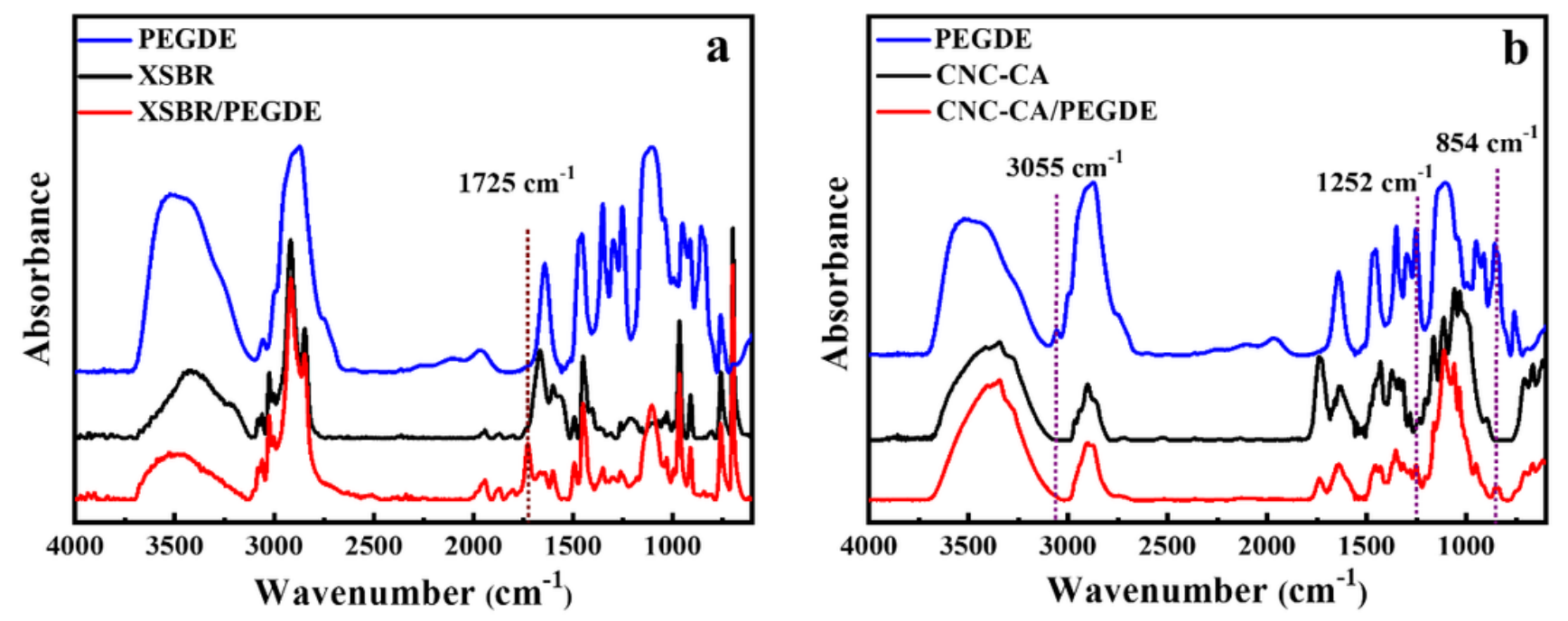
Figure 7

FTIR spectrum of (a) neat XSBR, PEGDE, XSBR/PEGDE and (b) CNC-CA, PEGDE, CNC-CA/PEGDE.

\section{Supplementary Files}

This is a list of supplementary files associated with this preprint. Click to download.

- Graphicalabstract.docx

- supportinginformation.docx

- schema1.png

- schema2.png 